\title{
Hierarchy Model of Application Framework ${ }^{(\mathbb{1}}$
}

\author{
Yin Shengbin ${ }^{1, ~ a ~}$, Gao Limin ${ }^{2, b}$ \\ ${ }^{1}$ Hebei University , Baoding, China, ${ }^{2}$ Hebei University ,Baoding, China \\ aysb@hbu.cn, bglm@hbu.cn
}

Keywords: Application Framework; Hierarchy model; Software Engineering; Component

\begin{abstract}
With the development of component technology, component granularity plays a very import role on the complexity of component composition. How to assemble the component of big granularity? How to make the component in the same domain integrated? Application framework brings the new era of software-reused technology. But how to construct application framework to make it higher reuse? According to the current research of application framework, the paper gives a hierarchy model; The model divides application framework into several layers. Different application framework in different layer has different solutions and the higher the application framework stands in, the lower the probability of change is.
\end{abstract}

\section{Introduction}

At present the research of the framework is placed in the stage of theories design. We described the framework abstractly in the theories and discovered most difference from the framework of our design to the framework of instantiation. To investigate its reason, when we are proceeding application system design, for adapting to the change of the customer requirement continuously, the framework are modified continually to adapt to the new request. Along with the time, the fixed part we defined in framework, namely "Frozen Spots"[1][2], may show some more change too. For avoiding this kind of circumstance, this paper put forward the hierarchy model of the application framework, the model divides the application framework into different layers. The framework of different hierarchy will be had different solution.

\section{The definition of the application framework}

Now we often adopt two definitions of the application framework in the below :(1)" framework is the reuse design of all or part of a system, usually make up of a set of abstract classes and cooperation of these classes"[4]. (2) "The framework is a system framework that can be made instantiation by developers"[5]. These two definitions describe the application framework in both sides, the former describes the framework from the angle of the design, putting forward the framework composing and the design mode; the latter describes the purpose of design.

From these two definitions we know:(1) The framework compartmentalizes the classes of application domain according to the system function, and defines main duties of every part of the system, and defines the synergic relation of objects and classes, moreover, limits the control flow too;(2) The framework includes the decision-making of main functional design of its application domain, it defines description and calling rule of the component[6][7]. So, the framework can be deemed to be an assembled component that can complete main function of system and be extensible. From this meaning, we can define the application framework as: It is a reusable system application model that can assemble necessary function of system and allow dynamic extension, and can be instantiation[8]. For better reuse the framework, the paper divides the framework into two kinds: The function framework and the structure framework. The function framework is a reuse model that can realize an indivisibility function. The structure framework is reuse model that can assemble a set of independent functions into a whole.

\footnotetext{
(1) This research funded by the project of Baoding science and technology, its number is 12ZG007
} 


\section{Hierarchy model of application framework}

The application framework is a generalizable description of system function; it is a "framework"[3] of system. At present, we often adopt a method to design the application framework; the method is to combine the analyses from top to down with the integration from down to top. This combined process actually not only is to divide the application framework into any layers, but also is abstract and generalization of system. Abstract in different level forms the framework in different layer. In other words, the framework at first formed is one of the highest reuse and can be extended system function agilely[3], then is to extend part of system function agilely, the rest may be deduced by analogy. As thus the framework of top layer generally is structure framework, in the middle layer is structure framework and function framework (Do not obviate the thing that direct call components, but these components must be independence completely, namely do not depend on other element), close relationship with the components on bottom layer is function framework. Such as Fig.1.

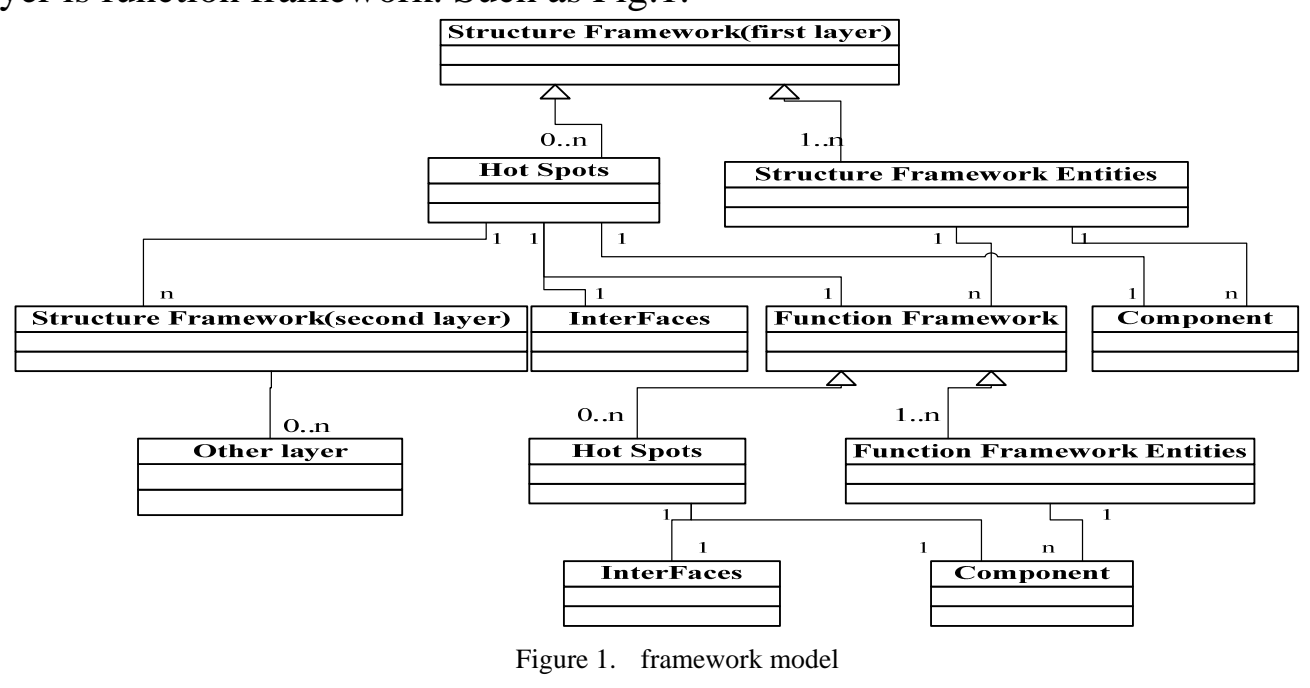

\section{A. Structure Framework Model}

The structure framework defines main function in system and extends system function dynamically through the interface, "Hot Spots". The structure framework does not actualize concrete system function, but realize it through calling the function entity. So we think from this meaning, the structure framework is an assembled tool and calling equipment. The assembled tool gathers system function together as a whole, and prescribe extense interface standard. Frameworks or components of according with the standard may be embedded in system. The calling equipment creates instance and initializes the parameters to actualize the system function. Its formalization describe is:

The structure framework is a group that is made up of five members:

Structure Framework= interface, property, functions register, function entity Call, Hot Spots $\}$

Among them:

Functions $=\{$ Items, Relations $\}$

Functions are made up of a set of function items of embedding in the framework and the Relations with entities that realize these functions.

Structure Framework framework_name

\{

Interfaces:

GUI ctrol Interface;

Interface of calling components;

Interface of calling subframework;

Properties:

Custom design Interface;

Initialization (); 
Get properties ();

Set properties ();

Functions register:

Item1:

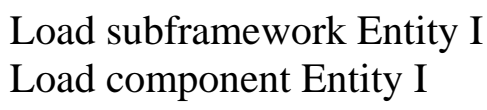

ItemN:

Load subframework Entity N

Load component Entity N

Function entity Call:

Item1:

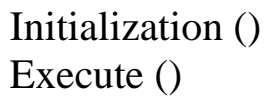

Hot Spots:

Other entities Interface ()

Entities self-adaption control process();

$\operatorname{Hook}()$;

\}

\section{B. Function Framework Model}

The function framework complete the function of calculation and control, at the same time it goes through some extense sockets to extend system function. The function framework usually not only includes a part of function entities, but also includes mutual basic establishment that helps to realize functions and extend function. Such as Fig. 2.

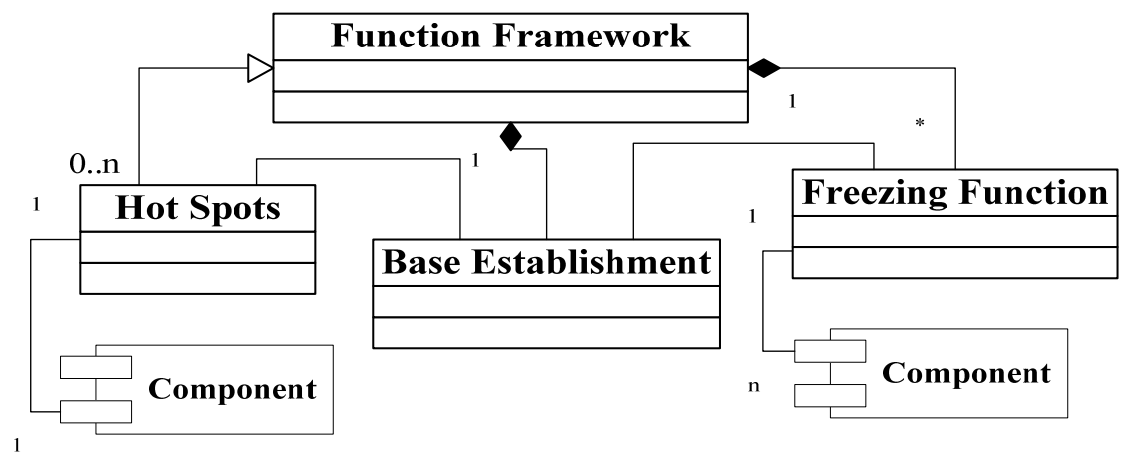

Figure 2. Function framework model

Its formalization describe is:

The function framework is a group that is made up of four members:

Function Framework $=\{$ Mutual Base Establishment, Frozen Function, Frozen Function Entity Call, Hot Spots

Function Framework framework_name

\{

Mutual Base Establishment: \{Controls (); Relative (); Display ()\}

Frozen Functions

Frozen Function Entity Call

Hot Spots

\}

C. Compare of the two frameworks' reuse degree

The structure framework' reuse degree is higher than the function framework's. Reason: the structure framework only put system functions together and do not relate to these functions' realization. If only frameworks or components accord with the standard of interface, they can 
embed in the system as plug -in. Usually the structure framework can be used as the main framework of application system.

The function framework' complexity and reuse degree have some change. Generally, higher complexity lower reuse degree, but this is not inverse ratio. When the function framework can cover appearing problems as soon as possible for realizing one function, its complexity is higher, but its reuse degree also increases.

Therefore, the structure framework suit for universal domain, but the function framework aims at the particular domain and its generalization is lower.

Such as Table 1.

TABLE I. COMPARE OF THE TWO FRAMEWORKS

\begin{tabular}{|l|l|l|}
\hline \multirow{2}{*}{ Category } & \multicolumn{2}{|c|}{ Project } \\
\cline { 2 - 3 } & structure framework & function framework \\
\hline Reuse model & black-box reuse & white-box reuse \\
\hline Reuse degree & high & low \\
\hline complexity & low & high \\
\hline suitable domain & common domain & particular domain \\
\hline extensibility & high & low \\
\hline
\end{tabular}

\section{Application instance}

When we developed the teaching evaluation system, we define the structure framework and the function framework starting with the analysis of the evaluation system (Such as Fig. 3). We create framework model basing on the analysis, and design framework tools to customize frameworks to adapt to different application system.

These frameworks have some characteristics, such as good encapsulation, reuse and low coupling degree, etc. Therefore this system possess the qualities of high-efficiency, expand easily and easy maintenance, etc.

\section{Conclusion}

The big granularity reuse of the framework make lower of system development and maintenance cost, make more quickly of developing velocity and reduce the developing persons. To divide framework into layers not only increases the framework reuse degree, but also strengthened with its adaptability, flexibility and extensibility. The design of the application framework oneself is a process that is circulation and iteration. It gets update and increase in the process that continuously applies with practice. We continuously improve the method of compartmentalizing layer of the framework too. In the future we need to farther study dynamic extension of the function framework. 


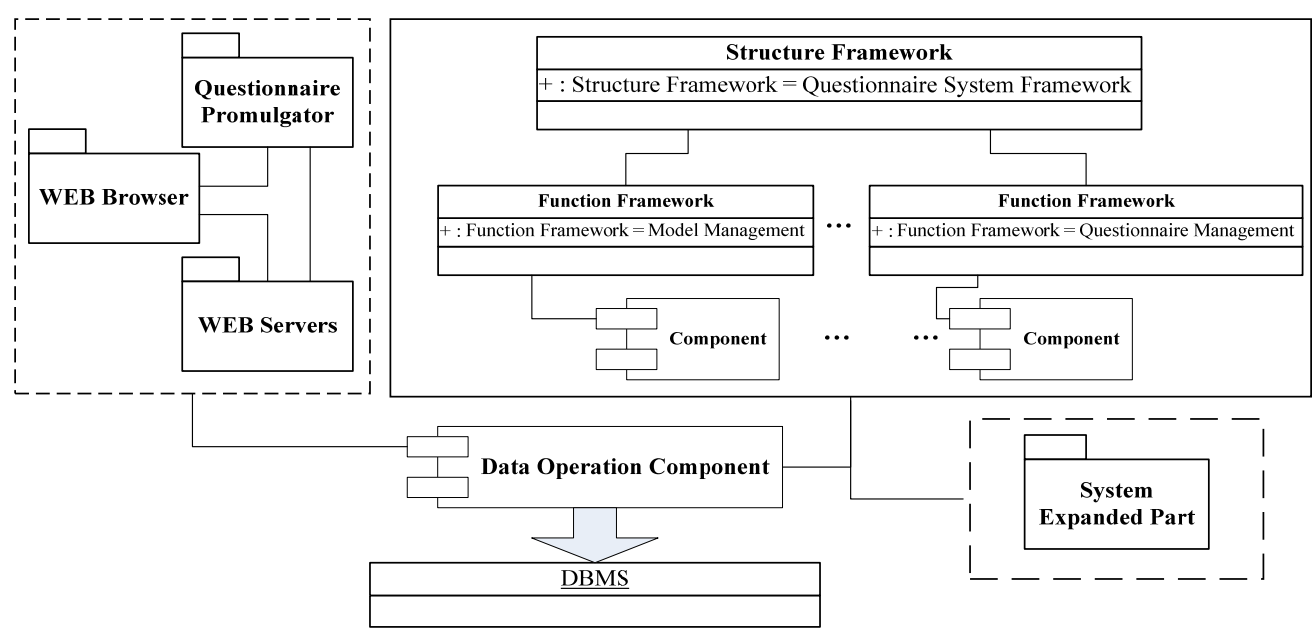

Figure 3. The teaching evaluation system structure

\section{References}

[1]. Johnson RE. Frameworks $=($ Components + Patterns $)[\mathrm{J}]$. Communications of the ACM, 1997,40(10):39 42.

[2]. Yang FQ, Mei H, Li KQ. Software reuse and software component technology [J]. Acta Electronica Sinca, 1999,27(2):68 75.

[3]. G.Succi,R.Wong,E.Liu,M.Smith,Supporting

Dynamic

Composition

of

Componens,ICAC04,IEEE,2004:85-86.

[4]. ShengBin Yin, XiaoFan Bian. Construct the Component of the immature realm Using Data Mining[C].Proceedings of 2004 International Conference on Machine Learning and Cybernetics,ShangHai,2004: 1509 1514.

[5]. BJOINER D. Software engineering 3 domains requirements and software design M. Berlin Springer 2006.

[6]. Luckham D,Vera J, Meldal S. Three concepts of system architecture[J]. Technical Report, CSL-TR-95-674, Stanford University, 1995.

[7]. Zhang SK, Zhang WJ, Chang X, Wang LF, Yang FQ. Building and assembling reusable components based on software architecture [J]. Journal of Software, 2001,12(9):1351 1359.

[8]. Garlan D, Monroe R, Wile D. ACME: An architectural interconnection language [J]. Technical Report, CMU-CS-95-219, Carnegie Mellon University, 1995. 